\title{
Hierarchy of efficiently computable and faithful lower bounds to quantum discord
}

\author{
Marco Piani ${ }^{1,2}$ \\ ${ }^{1}$ SUPA and Department of Physics, University of Strathclyde, Glasgow G4 ONG, UK \\ ${ }^{2}$ Department of Physics $\&$ Astronomy and Institute for Quantum Computing, \\ University of Waterloo, Waterloo, Ontario, N2L 3G1, Canada
}

\begin{abstract}
Quantum discord expresses a fundamental non-classicality of correlations that is more general than entanglement, but that, in its standard definition, is not easily evaluated. We derive a hierarchy of computationally efficient lower bounds to the standard quantum discord. Every non-trivial element of the hierarchy constitutes by itself a valid discord-like measure, based on a fundamental feature of quantum correlations: their lack of shareability. Our approach emphasizes how the difference between entanglement and discord depends on whether shareability is intended as a static property or as a dynamical process.

PACS numbers:
\end{abstract}

Correlations in quantum mechanics exhibit nonclassical features that include non-locality [1], steering [2], entanglement [3], and quantum discord [4], and that play a fundamental role in quantum information processing and quantum technologies [5], from quantum cryptography [6] to quantum metrology [7]. While both non-locality and steering are manifestations of entanglement, quantum discord is a more general form of quantumness of correlations that includes entanglement but goes beyond it. In fact, almost all distributed quantum states, including unentangled ones, exhibit discord [8]. The ubiquitousness of discord constitutes a motivation to understand it as much as possible - including its relation with entanglement - and also a call to fully elevate its study to an operational and quantitative level, since just certifying its presence may be considered of limited interest.

While several approaches to the quantification of discord have been proposed (see, e.g. [4, 9-22] and references therein), in this Letter we push forward a meaningful, reliable, and computationally friendly quantitative approach to the study of discord. Conceptually speaking, our approach offers a unifying way to understand both entanglement and discord in terms of shareability of correlations: entanglement is characterized by the impossibility of sharing correlations even in a static fashion [23]; discord is instead related to the impossibility of freely re-distributing correlations - a dynamical perspective. A unified view of quantum correlations based on a general notion of shareability is relevant in several contexts that deal with the distribution of quantum states: in quantum information processing, particularly in quantum cryptography $[24,25]$ and in entanglement distribution $[26,27]$; in quantum foundations, particularly in the study of the classical-to-quantum transition [28, 29]; in condensed-matter physics and quantum chemistry, particularly in relation to frustration phenomena [30-33] and to the quantum marginal problem $[34,35]$.

Quantum discord was introduced as the minimum amount of correlations, as measured by mutual informa- tion, that is necessarily lost in a local measurement of a bipartite quantum state [36, 37]. It is not easily evaluated [38, 39], and even general computable lower bounds are not known. In this Letter we provide a hierarchy of lower bounds to quantum discord that can be reliably computed numerically, and that have physical meaning, since they are based on 'impossibility features' related to the local manipulation of quantum correlations. Furthermore, our bounds satisfy the basic requests that should be imposed on any reasonable measure of quantum correlations [40, 41], hence they are valid discord-like quantifiers themselves.

One 'impossibility feature' associated to quantum discord relates to local broadcasting [42, 43]: correlations that exhibit quantum discord cannot be freely locally redistributed or broadcast, and discord can be interpreted as the asymptotic loss in correlations necessarily associated with such an attempt $[29,44]$. A very related 'impossibility feature' of discord deals with the 'local relocation' of quantum states by classical means, that is, via classical communication. The relation between the above two 'impossibility features' is given by the fact that quantum information becomes classical when broadcast to many parties [29, 45-47]. Besides their foundational interest, such features limit, for example, what a malicious eavesdropper can do in quantum cryptography [48, 49].

A result by Petz [50-52] implies that the ability to perfectly locally broadcast or transmit by classical means distributed quantum states reduces to the ability to perfectly locally broadcast or classically transmit correlations, as measured by mutual information [42, 43, 53], a feat possible - by definition - only in absence of discord. The consideration of the approximate classical transmission of an arbitrary quantum state, possibly exhibiting discord, has recently received renewed attention also thanks to a breakthrough result of Fawzi and Renner [54] (see also [55-58]) that generalizes the result by Petz. In [22] a discord-like quantifier was introduced, the surprisal of measurement recoverability; it quantifies how well - in terms of quantum fidelity - a distributed quan- 
tum state can be locally transmitted by classical means, and, thanks to [54], it directly translates into a lower bound to the standard quantum discord. Unfortunately, it is not easily computable either. In this Letter, by considering how well a quantum state can be locally broadcast, we generalize the surprisal of measurement recoverability to obtain a hierarchy of numerically computable bounds converging to it. The hierarchy exploits ideas used in the characterization and detection of entanglement via semidefinite programming (SDP) [59-61]. SDP optimization techniques [62] have found many other applications in quantum information (see, e.g., [63-69]), and recently have been used in the quantification of steering $[70,71]$. Here we extend the use of SDP techniques for the study of quantum correlations to quantum discord. This could be considered surprising, because the set of non-discordant states is not convex, contrary to, for example, the set of unentangled states. The key point is that our operational approach focuses on the local manipulation of correlations, rather than on their direct description, and we consider constraints on such a manipulation that are amenable to an SDP characterization.

Preliminaries.-We consider finite-dimensional systems. A quantum state corresponds to a positive semidefinite density matrix $\rho$ belonging to the space $L(\mathcal{H})$ of linear operators on a Hilbert space $\mathcal{H}$. The von Neumann entropy of $\rho$ is $S(\rho)=-\operatorname{Tr}(\rho \log \rho)$. We indicate by $\operatorname{Tr}_{\backslash X}$ a trace performed over every system except $X$, and $\log \equiv \log _{2}$. For the global state $\rho$ of a bi- or multi-partite system, we denote $S(X)_{\rho}=S\left(\rho_{X}\right)$, where $L\left(\mathcal{H}_{X}\right) \ni \rho_{X}=\operatorname{Tr}_{\backslash X}(\rho)$ is the reduced state of system $X$. The fidelity $F(\sigma, \rho)=\operatorname{Tr} \sqrt{\sqrt{\rho}} \sigma \sqrt{\rho}$ quantifies how similar two states $\rho$ and $\sigma$ are [5]. It holds $0 \leq F(\sigma, \rho) \leq 1$, with $F(\sigma, \rho)=1$ if and only if $\rho=\sigma$. In our analysis it will be important that the fidelity corresponds to the SDP optimization $[72,73]$

$$
\begin{array}{cl}
\text { maximize } & \frac{1}{2}\left(\operatorname{Tr}(X)+\operatorname{Tr}\left(X^{\dagger}\right)\right), \\
\text { subject to } & \left(\begin{array}{cc}
\rho & X \\
X^{\dagger} & \sigma
\end{array}\right) \geq 0 .
\end{array}
$$

Physical transformations are described by completelypositive trace-preserving linear maps - also called channels - on operators [5].

Separability and symmetric extensions.-A bipartite state $\rho_{A B}$ is separable (or unentangled) if it admits the decomposition

$$
\rho_{A B}^{\mathrm{sep}}=\sum_{b} p_{b}\left|\alpha_{b}\right\rangle\left\langle\left.\alpha_{b}\right|_{A} \otimes \mid \beta_{b}\right\rangle\left\langle\left.\beta_{b}\right|_{B},\right.
$$

for $\left\{p_{b}\right\}$ a probability distribution, and $\left|\alpha_{b}\right\rangle_{A}$ and $\left|\beta_{b}\right\rangle_{B}$ vector states for $A$ and $B$, respectively. The correlations present in a separable state can be explained classically, although they may exhibit a quantum behaviour, as we shall see. A bipartite state that is not separable is deemed entangled [74], and requires quantum communication or interaction to be generated.

Let $B^{k}=B_{1} B_{2} \ldots B_{k}$, with $B \simeq B_{1} \simeq B_{2} \simeq \ldots \simeq B_{k}$, i.e., the $B_{i}$ systems are 'copies' of the $B$ system. We say that $\rho_{A B^{k}}$ is a $k$-symmetric extension of $\rho_{A B}$ (on $B$ ) if:

(i) $\operatorname{Tr}_{\backslash A B_{i}}\left(\rho_{A B^{k}}\right)=: \rho_{A B_{i}}=\rho_{A B}$, for all $i=1, \ldots, k$;

(ii) $\rho_{A B^{k}}=V \rho_{A B^{k}} V^{\dagger}$ for any unitary $V$ that permutes the $B^{k}$ systems.

Because of the symmetry (ii), in (i) it is enough to consider the trace over all systems $B_{i}$ except one, e.g., $B_{1}$. If (i) and (ii) hold, it means the system $A$ can simultaneously share the same bipartite state $\rho_{A B}$ with $k$ different systems that are copies of $B$. If the stronger condition

(ii') $\rho_{A B^{k}}=\Pi_{B^{k}}^{+} \rho_{A B^{k}} \Pi_{B^{k}}^{+}$, with $\Pi_{B^{k}}^{+}$the projector onto the fully symmetric subspace $B_{+}^{k}$ of $B^{k}$,

holds, we say that $\rho_{A B^{k}}$ is a $k$-Bose-symmetric extension of $\rho_{A B}$ (on $B$ ).

A very important result in the study of quantum correlations is that only separable states like (2) admit $k$ symmetric extensions for all $k[61,75,76]$.

No local broadcasting. - The no-local-broadcasting theorem $[42,43]$ states that there exists a broadcasting channel $\Lambda_{B \rightarrow B_{1} B_{2}}$ such that $\Lambda_{B \rightarrow B_{1} B_{2}}\left[\rho^{A B}\right]$ is a 2 -symmetric copy of $\rho_{A B}$ if and only if $\rho^{A B}$ is quantum-classical, i.e., of the form

$$
\rho_{A B}^{\mathrm{qc}}=\sum_{b} p_{b} \rho_{b}^{A} \otimes|b\rangle\left\langle\left. b\right|_{B},\right.
$$

with $\{|b\rangle\}$ an orthonormal basis, and $\left\{p_{b}\right\}$ a probability distribution [97]. We notice that quantum-classical states form a strict subclass of the separable states (2); this means that, while the correlations of separable states can be explained in classical terms, their behaviour can bean in general is -non-classical.

If local broadcasting is possible, then it can be realized with a symmetric broadcasting channel, whose output is symmetric. More precisely, an arbitrary number $k$ of extensions can be obtained, simply by $|b\rangle \mapsto|b\rangle^{\otimes k}$, for $|b\rangle$ as in (3), i.e., with output in $B_{+}^{k}$ (see Fig. 1). Consider then Bose-symmetric broadcast maps $\Lambda_{B \rightarrow B_{+}^{k}}$, with output in the fully symmetric subspace, and the induced maps $\Lambda_{B}^{\mathrm{Sym}_{+}{ }^{(k)}}=\operatorname{Tr}_{\backslash B_{1}} \circ \Lambda_{B \rightarrow B_{+}^{k}}$ on $B$, where $\circ$ denotes composition. We say that any map $\Lambda_{B}^{\mathrm{Sym}_{+}{ }^{(k)}}$ that admits such a representation is $k$-Bose-symmetric extendible.

A measure-and-prepare channel-also called entanglement-breaking (EB) - acts according to [77] $\Lambda_{B}^{\mathrm{EB}}[\cdot]=\sum_{y} \operatorname{Tr}\left(M_{y}^{B} \cdot\right)\left|\beta_{y}\right\rangle\left\langle\left.\beta_{y}\right|_{B}\right.$, where $\left\{M_{y}^{B}\right\}$ is a positive-operator-valued measure (POVM) associate to a measurement, and the $\left|\beta_{y}\right\rangle_{B}$ 's are normalized vector states, associated to a $y$-dependent preparation. As the name suggests, entanglement-breaking maps are such that $\left(\operatorname{id}_{A} \otimes \Lambda_{B}^{\mathrm{EB}}\right)\left[\rho_{A B}\right]$ is separable for any $\rho_{A B}$. 


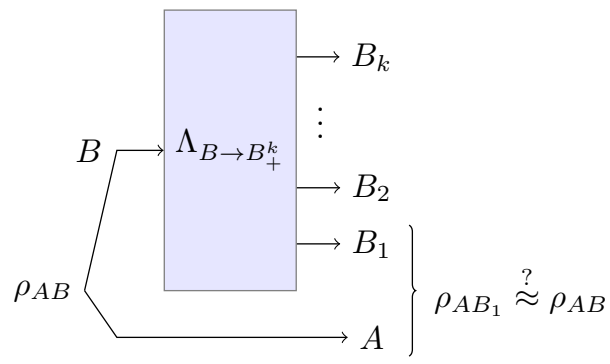

FIG. 1: Symmetric local broadcasting (colour online). A Bose$k$-symmetric broadcasting channel $\Lambda_{B \rightarrow B_{+}^{k}}$ has output in the fully symmetric subspace of $B^{k}=B_{1} B_{2} \ldots B_{k}$. How well $\rho_{A B_{1}}=\operatorname{Tr}_{\backslash A B_{1}}\left(\Lambda_{B \rightarrow B_{+}^{k}}\left[\rho_{A B}\right]\right)$ can approximate $\rho_{A B}$ depends on the classicality of correlations in $\rho_{A B}$.

For any $k$-Bose-symmetric extendible $\Lambda_{B}^{\mathrm{Sym}_{+}{ }^{(k)}}$ there is an entanglement-breaking map $\Lambda_{B}^{\mathrm{EB}}$ close to it [47, 78]. On the other hand, any entanglement-breaking map is $k$ Bose-symmetric extendible, for any $k$, since we can consider $\Lambda_{B \rightarrow B_{+}^{k}}[\cdot]=\sum_{y} \operatorname{Tr}\left(M_{y}^{B} \cdot\right)\left(\left|\beta_{y}\right\rangle\left\langle\left.\beta_{y}\right|^{\otimes k}\right)_{B_{+}^{k}}\right.$. One can then argue that the class of $k$-symmetric extendible channels, which we denote $\mathcal{L}^{\mathrm{Sym}_{+}{ }^{(k)}}$, converges to the set of entanglement-breaking channels, which we denote $\mathcal{L}^{\mathrm{EB}}$, for $k \rightarrow \infty[47,78]$.

Mutual information, recoverability, and discord-The mutual information

$$
I(A: B)_{\rho}=S(A)_{\rho}+S(B)_{\rho}-S(A B)_{\rho},
$$

is a fundamental measure of the total correlations present between $A$ and $B[5,79,80]$. The conditional mutual information is defined as [5]

$$
I(A: B \mid C)_{\rho}=I(A: B C)_{\rho}-I(A: C)_{\rho},
$$

i.e., it quantifies the decrease of correlations due to the loss of $B$. The strong subadditivity of the von Neumann entropy [81] says that $I(A: B \mid C)_{\rho} \geq 0$. A state $\rho=\rho_{A B C}$ such that $I(A: B \mid C)_{\rho}=0$ is said to form a Markov chain. Indeed, a result by Petz [50-52] ensures that there exists a 'recovery channel' $\mathcal{R}_{C \rightarrow B C}$ such that $\rho_{A B C}=$ $\mathcal{R}_{C \rightarrow B C}\left[\rho_{A C}\right]$. Fawzi and Renner proved [54] that, more in general, for any tripartite state $\rho_{A B C}$, there exists a recovery channel $\mathcal{R}_{C \rightarrow B C}$ such that (see also [55-58])

$$
F\left(\mathcal{R}_{C \rightarrow B C}\left[\rho_{A C}\right], \rho_{A B C}\right) \geq 2^{-\frac{1}{2} I(A: B \mid C)_{\rho}},
$$

that is, the smaller the decrease of correlations due to the loss of $B$, the better - in fidelity terms - $\rho_{A B C}$ can be recovered from operating on $C$ alone.

The discord $D(A: B)_{\rho}$ of $\rho=\rho_{A B}$ between $A$ and $B$ with measurement on $B$ is the minimal loss of correlations that occurs when $B$ is measured. Let $\mathcal{M}_{B \rightarrow Y}[\cdot]=\sum_{y} \operatorname{Tr}\left(M_{y}^{B} \cdot\right)|y\rangle\left\langle\left. y\right|_{Y}\right.$ be a generic measurement map, where $\left\{M_{y}\right\}$ is a POVM, and $\{|y\rangle\}$ is an orthonormal basis. Then $[36,37]$

$$
D(A: \underline{B})_{\rho}:=\min _{\mathcal{M}_{B \rightarrow Y}}\left(I(A: B)_{\rho}-I(A: Y)_{\mathcal{M}_{B \rightarrow Y}[\rho]}\right) .
$$

Discord vanishes only for the quantum-classical states $(3)[43,53]$. Like any other channel, a measurement map can be seen as the result of the coherent interaction with an environment, followed by the tracing out of some subsystem [5], a 'loss' that one can try to 'undo' via a recovery channel. In the case of a (local) measurement, the recovery correspond to a preparation, and the combination of measurement and recovery is an entanglement-breaking map $\Lambda^{\mathrm{EB}}$ [22]. Thus, putting together the result (4) of Fawzi-Renner with the defining notion of discord, one arrives at [22]

$$
\sup _{\Lambda^{\mathrm{EB}} \in \mathcal{L}^{\mathrm{EB}}} F\left(\Lambda_{B}^{\mathrm{EB}}\left[\rho_{A B}\right], \rho_{A B}\right) \geq 2^{-\frac{1}{2} D(A: \underline{B})} .
$$

Defining the surprisal of measurement recoverability [22]

$$
D_{F, \mathcal{L}^{\mathrm{EB}}}(A: \underline{B}):=-\log \sup _{\Lambda^{\mathrm{EB}} \in \mathcal{L}^{\mathrm{EB}}} F^{2}\left(\Lambda_{B}^{\mathrm{EB}}\left[\rho_{A B}\right], \rho_{A B}\right),
$$

one can cast $(5)$ as $D_{F, \mathcal{L}^{\mathrm{EB}}}(A: \underline{B}) \leq D(A: \underline{B})$. The quantity $D_{F, \mathcal{L}^{\operatorname{EB}}}(A: \underline{B})$ measures the inevitable disturbance introduced by manipulating locally (on $B$ ) the state $\rho_{A B}$, through measurement and (re-)preparation. This can be generalized to any non-trivial (local) manipulation (see also [20]), i.e., one can consider

$$
D_{F, \mathcal{L}}(A: \underline{B}):=-\log \sup _{\Lambda \in \mathcal{L}} F^{2}\left(\Lambda_{B}\left[\rho_{A B}\right], \rho_{A B}\right),
$$

for a generic class of channels $\mathcal{L}$. This corresponds to quantifying the best fidelity that can be achieved in the transmission of the $B$ component of $\rho_{A B}$, through a channel that belongs to $\mathcal{L}$. If $\mathcal{L}^{\mathrm{EB}} \subseteq \mathcal{L}$, it necessarily holds $D_{F, \mathcal{L}}(A: \underline{B}) \leq D_{F, \mathcal{L}^{\mathrm{EB}}}(A: \underline{B}) \leq D(A: \underline{B})$. In this Letter we focus on $\mathcal{L}=\mathcal{L}^{\mathrm{Sym}_{+}(k)}$, that is, we consider the disturbance induced by channels that admit $k$ extensions. Equivalently, we can say we are considering how well one can broadcast the $B$ component of $\rho_{A B}$ to $k$ receivers, which is a relevant problem in quantum communication and in quantum cryptography, as this kind of 'symmetric cloning' (particularly for $k=2$ ) is a typical attack that an eavesdropper can perform [48, 49]. Since $\mathcal{L}^{\mathrm{EB}} \subseteq \mathcal{L}^{\mathrm{Sym}_{+}(k)}$, it follows

$$
D_{F, \mathcal{L}^{\mathrm{Sym}_{+}(k)}}(A: \underline{B}) \leq D_{F, \mathcal{L}^{\mathrm{EB}}}(A: \underline{B}) \leq D(A: \underline{B}),
$$

for all $k \geq 2$. Furthermore, one proves [78] $\lim _{k \rightarrow \infty} D_{F, \mathcal{L}^{\mathrm{Sym}_{+}(k)}}(A: \underline{B})=D_{F, \mathcal{L}^{\mathrm{EB}}}(A: \underline{B})$.

An SDP hierarchy of discord-like measures.-The Choi-Jamiołkowski isomorphism $[82,83]$ is a one-to-one correspondence between linear maps and linear operators. Through the isomorphism, specific properties of the linear maps are associated with specific properties of linear operators. The computation of the surprisal of measurement recoverability requires a difficult optimization over entanglement-breaking channels. Relaxing the problem, we maximize the fidelity between $\rho=\rho_{A B}$ 


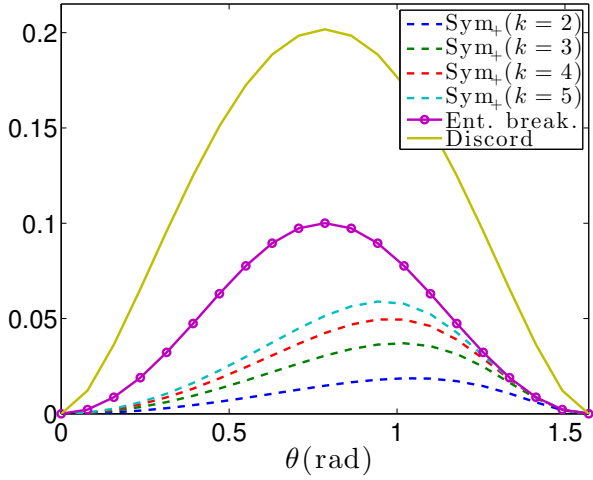

FIG. 2: A hierarchy of lower bounds to quantum discord (colour online). Let $\rho_{A B}(\theta)=\frac{1}{2}|0\rangle\left\langle\left. 0\right|_{A} \otimes \mid \psi_{0}(\theta)\right\rangle\left\langle\left.\psi_{0}(\theta)\right|_{B}+\right.$ $\frac{1}{2}|1\rangle\left\langle\left. 1\right|_{A} \otimes \mid \psi_{1}(\theta)\right\rangle\left\langle\left.\psi_{1}(\theta)\right|_{B}\right.$, with $\left.\mid \psi_{a}(\theta)\right\rangle=\cos (\theta / 2)|0\rangle+$ $(-1)^{a} \sin (\theta / 2)|1\rangle, a=0,1$, for $\theta \in[0, \pi / 2]$. From bottom to top, we plot $D_{F, \mathcal{L}^{\mathrm{Sym}_{+}}{ }^{(k)}}$ for $k=2,3,4,5$ (dashed lines), $D_{F, \mathcal{L}^{\mathrm{EB}}}$ (line with circles), as calculated via SDP, and the discord proper $D$ (on $B$ ) (solid line) as calculated in [84, 85]. Any element in the hierarchy certifies quantitatively that the state $\rho_{A B}(\theta)$ is classical on $B$ only for $\theta=0, \pi / 2$.

and $\sigma=\left(\operatorname{id}_{A} \otimes \Lambda_{B}^{\mathrm{Sym}_{+}{ }^{(k)}}\right)\left[\rho_{A B}\right]$, where $\Lambda_{B}^{\mathrm{Sym}_{+}(k)}$ is a $k$-extendible map. Using the Choi-Jamiolkowski isomorphism and the SDP expression of the fidelity (1), this can be cast as the SDP optimization [78]:

$$
\begin{array}{ll}
\max & \frac{1}{2}\left(\operatorname{Tr}(X)+\operatorname{Tr}\left(X^{\dagger}\right)\right) \\
\text { s. t. } & \left(\begin{array}{cc}
\rho_{A B} & X \\
X^{\dagger} & \operatorname{Tr} \backslash A B_{1} \\
& \left(W_{B B^{k}}^{\Gamma_{B}} \rho_{A B}\right)
\end{array}\right) \geq 0 \\
& W_{B B^{k}} \geq 0, W_{B}=\mathbb{1}_{B}, W_{B B^{k}}=\Pi_{B^{k}}^{+} W_{B B^{k}} \Pi_{B^{k}}^{+} .
\end{array}
$$

Eqs. (7a) and (7b) directly correspond to Eqs. (1a) and (1b); Eqs. (7c) constrain the Choi-Jamiołkowski operator $W_{B B^{k}}$ so that, in $(7 \mathrm{~b}), \operatorname{Tr}_{\backslash A B_{1}}\left(W_{B B^{k}}^{\Gamma_{B}} \rho_{A B}\right)=$ $\left(\operatorname{id}_{A} \otimes \Lambda_{B}^{\mathrm{Sym}_{+}(k)}\right)\left[\rho_{A B}\right]$, with $\Lambda_{B}^{\mathrm{Sym}_{+}(k)}$ the $k$-extendible channel isomorphic to $W_{B B_{1}}$. We already know that $D_{F, \mathcal{L}^{\mathrm{Sym}_{+}(k)}}(A: \underline{B})$ converges to $D_{F, \mathcal{L}^{\mathrm{EB}}}(A: \underline{B})$, for $k \rightarrow \infty$. One further checks that it does so by increasing monotonically with $k$ [78]. The no-local-broadcasting theorem ensures that already the lowest non-trivial element of the hierarchy $(k=2)$ is strictly positive for any state that is not classical on $B$. Furthermore, each $D_{F, \mathcal{L}^{\mathrm{Sym}_{+}}{ }^{(k)}}$ constitutes a well-behaved measure of the general quantumness of correlations [40, 41].

We can choose to impose additional properties on the class $\mathcal{L}$ of channels considered, so to, e.g., make them better approximate entanglement-breaking channels, and even calculate the exact numerical value for the surprisal of measurement recoverability in the case where the broadcast system is a qubit [78]. We implemented (7) in MATLAB [86], making use of CVX [87, 88] and other tools publicly available [89, 90]; see Figure 2 for numerical results.

Discord, entanglement, and symmetric extensions.Our approach, based on an SDP hierarchy dealing with symmetric extensions, is inspired by the one used to verify entanglement [59, 60] (see also [63] for applications to the extendability of channels). It suggests an illuminating and unifying point of view on entanglement and discord in terms of symmetric extensions. Entanglement limits how well a state can be approximated by a state admitting a $k$-symmetric extension [61, 91]; only separable states admits such extensions for all $k \geq 2$. Discord instead limits how well a state can be locally transformed into a $k$-symmetric extension of itself: only discord-free states can be perfectly locally broadcast, for any $k \geq 2$. While entanglement can be exactly characterized only in the limit $k \rightarrow \infty$, discord already is in the case $k=2$-this is the content of the no-local-broadcasting theorem $[42,43]$.

Conclusions. - We introduced a hierarchy of discordlike quantifiers, based on how well a quantum state $\rho_{A B}$ can be locally broadcast. In the limit where we consider infinite output copies of $B$, we end up addressing the question of how well the $B$ part of $\rho_{A B}$ can be transmitted through a measure-and-prepare process. Each non-trivial discord-like quantifier in our hierarchy is non-vanishing for all the states that are not classical on $B$, and corresponds to an SDP optimization problem. It can be reliably and efficiently computed numerically, because of the polynomial scaling of the parameters of the SDP in the dimensions of the systems involved (for fixed number of copies) and in the number of copies (for fixed dimensions) $[59,60]$. Each element of the hierarchy has a clear physical meaning in itself and satisfies the basic properties to be expected for a meaningful discordlike quantifier, but it also constitutes a lower bound to the standard quantum discord $[36,37]$. In the case of a qubit-qudit system, with measurement on the qubit, a tailored SDP program outputs the surprisal of measurement recoverability of Ref. [22], and thus the best possible lower bound to standard quantum discord based on the breakthrough result about quantum Markov chains of Fawzi and Renner [54]. Our approach sheds light on the conceptual relation between entanglement, discord, and shareability, with potential applications in diverse fields like quantum information processing, quantum foundations, and many-body physics. Two questions that we leave open for future work regard the possible extension of our approach to the multipartite case and to infinitedimensional systems; we point out that the consideration of a discord-like quantifier with local measurements on many parties [42] poses a challenge in approximating the local measure-and-prepare channels in a way amenable to an SDP, since it is naturally non-linear in the unknown local operations [92].

Acknowledgements - I acknowledge support from 
NSERC. This project has received funding from the European Unions Horizon 2020 research and innovation programme under the Marie Skłodowska-Curie grant agreement No 661338. I would like to thank K. P. Seshadreesan, M. M. Wilde, and A. Streltsov for discussions, and G. Adesso and J. Eisert for useful correspondence.

[1] N. Brunner, D. Cavalcanti, S. Pironio, V. Scarani, and S. Wehner, Rev. Mod. Phys. 86, 419 (2014), URL http: //link.aps.org/doi/10.1103/RevModPhys.86.419.

[2] H. M. Wiseman, S. J. Jones, and A. C. Doherty, Phys. Rev. Lett. 98, 140402 (2007), URL http://link.aps. org/doi/10.1103/PhysRevLett.98.140402.

[3] R. Horodecki, P. Horodecki, M. Horodecki, and K. Horodecki, Rev. Mod. Phys. 81, 865 (2009), URL http://link.aps.org/doi/10.1103/RevModPhys. 81.865.

[4] K. Modi, A. Brodutch, H. Cable, T. Paterek, and V. Vedral, Rev. Mod. Phys. 84, 1655 (2012).

[5] M. A. Nielsen and I. L. Chuang, Quantum computation and quantum information (Cambridge University Press, 2010).

[6] N. Gisin, G. Ribordy, W. Tittel, and H. Zbinden, Rev. Mod. Phys. 74, 145 (2002), URL http://link.aps.org/ doi/10.1103/RevModPhys.74.145.

[7] V. Giovannetti, S. Lloyd, and L. Maccone, Nature Photonics 5, 222 (2011).

[8] A. Ferraro, L. Aolita, D. Cavalcanti, F. Cucchietti, and A. Acin, Phys. Rev. A 81, 052318 (2010).

[9] S. Luo, Phys. Rev. A 77, 022301 (2008).

[10] K. Modi, T. Paterek, W. Son, V. Vedral, and M. Williamson, Phys. Rev. Lett. 104, 080501 (2010).

[11] B. Dakić, V. Vedral, and Č. Brukner, Phys. Rev. Lett. 105, 190502 (2010).

[12] S. Luo and S. Fu, Phys. Rev. A 82, 034302 (2010).

[13] A. Streltsov, H. Kampermann, and D. Bruß, Phys. Rev. Lett. 106, 160401 (2011).

[14] M. Piani, S. Gharibian, G. Adesso, J. Calsamiglia, P. Horodecki, and A. Winter, Phys. Rev. Lett. 106, 220403 (2011).

[15] D. Girolami and G. Adesso, Phys. Rev. Lett. 108, 150403 (2012).

[16] M. Piani and G. Adesso, Phys. Rev. A 85, 040301 (2012).

[17] F. Paula, T. R. de Oliveira, and M. Sarandy, Phys. Rev. A 87, 064101 (2013).

[18] L. Chang and S. Luo, Phys. Rev. A 87, 062303 (2013).

[19] D. Girolami, A. M. Souza, V. Giovannetti, T. Tufarelli, J. G. Filgueiras, R. S. Sarthour, D. O. Soares-Pinto, I. S. Oliveira, and G. Adesso, Phys. Rev. Lett. 112, 210401 (2014).

[20] M. Piani, V. Narasimhachar, and J. Calsamiglia, New J. Phys. 16, 113001 (2014), URL http://stacks.iop.org/ $1367-2630 / 16 / i=11 / a=113001$.

[21] K. P. Seshadreesan, M. Berta, and M. M. Wilde, Journal of Physics A: Mathematical and Theoretical 48, 395303 (2015), URL http://stacks . iop.org/1751-8121/48/i= $39 / a=395303$.

[22] K. P. Seshadreesan and M. M. Wilde, Phys. Rev. A
92, 042321 (2015), URL http://link.aps.org/doi/10. 1103/PhysRevA.92.042321.

[23] M. Seevinck, Quantum information processing 9, 273 (2010).

[24] N. Gisin, G. Ribordy, W. Tittel, and H. Zbinden, Reviews of modern physics 74, 145 (2002).

[25] S. Pirandola, Scientific reports 4, 6956 (2014).

[26] T. Chuan, J. Maillard, K. Modi, T. Paterek, M. Paternostro, and M. Piani, Phys. Rev. Lett. 109, 070501 (2012).

[27] A. Streltsov, H. Kampermann, and D. Bruß, Phys. Rev. Lett. 108, 250501 (2012).

[28] W. H. Zurek, Nature Physics 5, 181 (2009).

[29] F. G. Brandão, M. Piani, and P. Horodecki, Nature Communications 6, 7908 (2015).

[30] K. Binder and A. P. Young, Rev. Mod. Phys. 58, 801 (1986).

[31] H. T. Diep, Frustrated spin systems (World Scientific, Singapore, 2005).

[32] C. M. Dawson and M. A. Nielsen, Phys. Rev. A 69, 052316 (2004).

[33] U. Marzolino, S. M. Giampaolo, and F. Illuminati, Phys. Rev. A 88, 020301 (2013), URL http://link.aps.org/ doi/10.1103/PhysRevA.88.020301.

[34] C. Schilling, The quantum marginal problem (World Scientific, Singapore, 2014).

[35] L. Chen, O. Gittsovich, K. Modi, and M. Piani, Physical Review A 90, 042314 (2014).

[36] H. Ollivier and W. H. Zurek, Phys. Rev. Lett. 88, 017901 (2001).

[37] L. Henderson and V. Vedral, J. Phys. A: Math. Gen. 34, 6899 (2001).

[38] D. Girolami and G. Adesso, Phys. Rev. A 83, 052108 (2011), URL http://link.aps.org/doi/10. 1103/PhysRevA . 83.052108.

[39] Y. Huang, New J. Phys. 16, 033027 (2014), URL http: //stacks . iop.org/1367-2630/16/i=3/a=033027.

[40] A. Brodutch and K. Modi, Quant. Inf. Comp. 12, 0721 (2012).

[41] M. Piani, Phys. Rev. A 86, 034101 (2012).

[42] M. Piani, P. Horodecki, and R. Horodecki, Phys. Rev. Lett. 100, 090502 (2008).

[43] S. Luo and W. Sun, Phys. Rev. A 82, 012338 (2010).

[44] A. Streltsov and W. H. Zurek, Phys. Rev. Lett. 111, 040401 (2013).

[45] J. Bae and A. Acín, Phys. Rev. Lett. 97, 030402 (2006).

[46] G. Chiribella and G. M. D'Ariano, Phys. Rev. Lett. 97, 250503 (2006).

[47] G. Chiribella, in Theory of Quantum Computation, Communication, and Cryptography (Springer, 2011), vol. 6519, pp. 9-25.

[48] N. Gisin and B. Huttner, Physics Letters A 228, 13 (1997).

[49] A. Ferenczi and N. Lütkenhaus, Phys. Rev. A 85, 052310 (2012), URL http://link.aps.org/doi/10. 1103/PhysRevA.85.052310.

[50] D. Petz, Comm. Math. Phys. 105, 123 (1986).

[51] D. Petz, The Quarterly Journal of Mathematics 39, 97 (1988).

[52] P. Hayden, R. Jozsa, D. Petz, and A. Winter, Comm. Math. Phys. 246, 359 (2004).

[53] M. Hayashi, Quantum Information (Springer, 2006).

[54] O. Fawzi and R. Renner, Communications in Mathematical Physics 340, 575 (2015).

[55] F. G. Brandão, A. W. Harrow, J. Oppenheim, and 
S. Strelchuk, Phys. Rev. Lett. 115, 050501 (2015).

[56] M. Berta and M. Tomamichel, arXiv preprint arXiv:1502.07973 (2015).

[57] M. M. Wilde, Proceedings of the Royal Society of London A: Mathematical, Physical and Engineering Sciences 471 (2015), ISSN 1364-5021, http://rspa.royalsocietypublishing.org/content/471/2182/201 URL http://rspa.royalsocietypublishing.org/ content/471/2182/20150338.

[58] D. Sutter, O. Fawzi, and R. Renner, arXiv preprint arXiv:1504.07251 (2015).

[59] A. C. Doherty, P. A. Parrilo, and F. M. Spedalieri, Phys. Rev. Lett. 88, 187904 (2002), URL http://link.aps. org/doi/10.1103/PhysRevLett.88.187904.

[60] A. C. Doherty, P. A. Parrilo, and F. M. Spedalieri, Phys. Rev. A 69, 022308 (2004), URL http://link.aps.org/ doi/10.1103/PhysRevA.69.022308.

[61] A. C. Doherty, J. Phys. A: Math. Th. 47, 424004 (2014), URL http://stacks.iop.org/1751-8121/47/i= $42 / a=424004$.

[62] S. Boyd and L. Vandenberghe, Convex optimization (Cambridge University Press, 2009).

[63] M. L. Nowakowski and P. Horodecki, J. Phys. A: Math. Th. 42, 135306 (2009), URL http://stacks.iop.org/ $1751-8121 / 42 / i=13 / a=135306$.

[64] R. Jain, Z. Ji, S. Upadhyay, and J. Watrous, Journal of the ACM (JACM) 58, 30 (2011).

[65] J. Kempe, O. Regev, and B. Toner, SIAM Journal on Computing 39, 3207 (2010).

[66] M. Navascués, S. Pironio, and A. Acín, Phys. Rev. Lett. 98, 010401 (2007), URL http://link.aps .org/doi/10. 1103/PhysRevLett.98.010401.

[67] J. Watrous, Theory of Computing 5 (2009).

[68] N. Johnston and D. W. Kribs, J. Math. Phys. 51, 082202 (2010).

[69] J. Eisert, F. Brandao, and K. Audenaert, New J. Phys. 9, 46 (2007), URL http://stacks.iop.org/1367-2630/ $9 / i=3 / a=046$.

[70] P. Skrzypczyk, M. Navascués, and D. Cavalcanti, Phys. Rev. Lett. 112, 180404 (2014), URL http://link.aps. org/doi/10.1103/PhysRevLett.112.180404.

[71] M. Piani and J. Watrous, Phys. Rev. Lett. 114, 060404 (2015), URL http://link.aps.org/doi/10. 1103/PhysRevLett.114.060404.

[72] N. Killoran, Ph.D. thesis, University of Waterloo (2012).

[73] J. Watrous, arXiv preprint arXiv:1207.5726 (2012).

[74] R. F. Werner, Phys. Rev. A 40, 4277 (1989), URL http: //link.aps.org/doi/10.1103/PhysRevA.40.4277.

[75] M. Fannes, J. Lewis, and A. Verbeure, Lett. Math. Phys. 15, 255 (1988).
[76] G. Raggio and R. Werner, Helvetica Physica Acta 62, 980 (1989).

[77] M. Horodecki, P. W. Shor, and M. B. Ruskai, Rev. Math. Phys. 15, 629 (2003).

[78] See Supplementary Material at ???, which refers also to [5, 93-96].

150338.fGtquidfinan, S. Popescu, and A. Winter, Phys. Rev. A 72, 032317 (2005), URL http://link.aps.org/doi/10. 1103/PhysRevA.72.032317.

[80] M. M. Wilde, Quantum information theory (Cambridge University Press, 2013).

[81] E. H. Lieb and M. B. Ruskai, J. Math. Phys. 14, 1938 (1973).

[82] M.-D. Choi, Lin. Alg. Appl. 10, 285 (1975).

[83] A. Jamiołkowski, Rep. Math. Phys. 3, 275 (1972).

[84] C. Fuchs, in Quantum Communication, Computing, and Measurement 2, edited by P. Kumar, G. D'Ariano, and O. Hirota (Springer US, 2002), pp. 11-16, ISBN 978-0-306-46307-5, URL http://dx.doi.org/10.1007/ 0-306-47097-7_2.

[85] Y. Yao, J.-Z. Huang, X.-B. Zou, and Z.-F. Han, Quantum Information Processing pp. 1-12 (2013).

[86] MATLAB, R2014a (The MathWorks Inc., Natick, Massachusetts, 2014).

[87] M. Grant and S. Boyd, CVX: Matlab software for disciplined convex programming, version 2.1, http://cvxr. com/cvx (2014).

[88] M. Grant and S. Boyd, in Recent Advances in Learning and Control, edited by V. Blondel, S. Boyd, and H. Kimura (Springer-Verlag Limited, 2008), Lecture Notes in Control and Information Sciences, pp. 95-110, http://stanford.edu/ boyd/graph_dcp.html.

[89] N. Johnston, QETLAB: A MATLAB toolbox for quantum entanglement, version 0.7, http://qetlab.com (2015).

[90] T. Cubitt, Maths code, http://www.dr-qubit.org/ matlab.php (2015).

[91] M. Navascués, M. Owari, and M. B. Plenio, Physical Review A 80, 052306 (2009).

[92] M. Piani, in preparation.

[93] A. Uhlmann, Rep. Math. Phys. 9, 273 (1976).

[94] C. A. Fuchs and J. Van De Graaf, Information Theory, IEEE Transactions on 45, 1216 (1999).

[95] P. Horodecki, M. Horodecki, and R. Horodecki, J. Mod. Opt. 47, 347 (2000).

[96] M. Horodecki, P. Horodecki, and R. Horodecki, Phys. Lett. A 223, 1 (1996).

[97] Here we focus on one-sided local broadcasting [43], rather than two-sided local broadcasting [42]. 


\title{
Supplementary Information for "Hierarchy of efficiently computable and faithful lower bounds to quantum discord"
}

\author{
Marco Piani ${ }^{1,2}$ \\ ${ }^{1}$ SUPA and Department of Physics, University of Strathclyde, Glasgow G4 ONG, UK \\ ${ }^{2}$ Department of Physics $\&$ Astronomy and Institute for Quantum Computing, \\ University of Waterloo, Waterloo, Ontario, N2L 3G1, Canada
}

In this Supplementary Information we provide the detailed proofs for the claims in the main text. Please refer to the main text for definitions and notation.

\section{CONVERGENCE OF THE HIERARCHY OF DISCORD-LIKE MEASURES}

A measure of similarity of states with an operational interpretation is the trace distance [1]

$$
\Delta(\sigma, \rho)=\frac{1}{2}\|\sigma-\rho\|_{1},
$$

where $\|\xi\|_{1}=\operatorname{Tr}\left(\sqrt{\xi^{\dagger} \xi}\right)$. It holds $0 \leq \Delta(\sigma, \rho) \leq 1$, and $1-F(\sigma, \rho) \leq \Delta(\sigma, \rho) \leq \sqrt{1-F^{2}(\sigma, \rho)}$, where $F$ is the fidelity [2].

Lemma 1. [3] Let $\Lambda^{\text {Sym }_{+}{ }^{(k)}}$ be a $k$-Bose-extendible channel acting on a space $B$ of dimension $|B|$. There is an entanglement-breaking channel $\Lambda^{E B}$ on $B$ such that

$$
\sup _{\rho_{A B}} \Delta\left(\Lambda_{B}^{S y m_{+}(k)}\left[\rho_{A B}\right], \Lambda_{B}^{E B}\left[\rho_{A B}\right]\right) \leq \frac{|B|}{k} .
$$

Given also that every entanglement-breaking channel $\Lambda_{B}^{\mathrm{EB}}$ is automatically $k$-Bose-extendible, Lemma 1 implies that the class of $k$-Bose-extendible channels tends to the class of entanglement-breaking maps for $k \rightarrow \infty$. Notice that we are here interested in channels with the same (and fixed) input/output dimension $|B|$, but we let $k$ vary.

Lemma 2. Consider any three mixed states $\rho, \sigma$, and $\tau$. It holds,

$$
\begin{aligned}
|F(\rho, \sigma)-F(\tau, \sigma)| & \leq \sqrt{2} \sqrt{1-F(\tau, \rho)} \\
& \leq \sqrt{2} \sqrt{\Delta(\tau, \rho)} .
\end{aligned}
$$

Proof. Fix an arbitrary purification $\left|\psi_{\rho}\right\rangle$, and choose purifications $\left|\psi_{\sigma}\right\rangle$ and $\left|\psi_{\tau}\right\rangle$ such that $\left\langle\psi_{\rho} \mid \psi_{\sigma}\right\rangle=F(\rho, \sigma)$ and $\left\langle\psi_{\rho} \mid \psi_{\tau}\right\rangle=F(\rho, \tau)$. This is always possible because of Uhlmann's theorem [1, 4] and by choosing properly phases.

Then,

$$
\begin{aligned}
F(\rho, \sigma) & =\left|\left\langle\psi_{\rho} \mid \psi_{\sigma}\right\rangle\right| \\
& =\mid\left(\left(\left\langle\psi_{\rho}\left|-\left\langle\psi_{\tau}\right|\right)+\left\langle\psi_{\tau}\right|\right)\left|\psi_{\sigma}\right\rangle \mid\right.\right. \\
& \leq\left|\left\langle\psi_{\tau} \mid \psi_{\sigma}\right\rangle\right|+\mid\left(\left\langle\psi_{\rho}\left|-\left\langle\psi_{\tau}\right|\right) \mid \psi_{\sigma}\right\rangle \mid\right. \\
& \leq F(\tau, \sigma)+\sqrt{\left(\left\langle\psi_{\rho}\left|-\left\langle\psi_{\tau}\right|\right)\left(\left|\psi_{\rho}\right\rangle-\left|\psi_{\tau}\right\rangle\right)\right.\right.} \\
& =F(\tau, \sigma)+\sqrt{2-\left\langle\psi_{\tau} \mid \psi_{\rho}\right\rangle-\left\langle\psi_{\rho} \mid \psi_{\tau}\right\rangle} \\
& =F(\tau, \sigma)+\sqrt{2} \sqrt{1-F(\tau, \rho)} \\
& \leq F(\tau, \sigma)+\sqrt{2} \sqrt{\Delta(\tau, \rho)} .
\end{aligned}
$$

The first inequality is just the triangle inequality for the absolute value. The second inequality is due to the fact that the fidelity between two states is the maximum overlap of any two purifications of the states [1, 4]. The last inequality is due to the standard relation $1-F(\tau, \rho) \leq \Delta(\tau, \rho)[1,2]$. 
We want to prove that $\lim _{k \rightarrow \infty} D_{F, \mathcal{L}^{\mathrm{Sym}}+(k)}(A: \underline{B})=D_{F, \mathcal{L}^{\mathrm{EB}}}(A: \underline{B})$. Given that

$$
D_{F, \mathcal{L}}(A: \underline{B}):=-\log \sup _{\Lambda \in \mathcal{L}} F^{2}\left(\Lambda_{B}\left[\rho_{A B}\right], \rho_{A B}\right),
$$

for a generic class of channels $\mathcal{L}$, and given that every entanglement-breaking channel is also $k$-Bose-extendible for any $k$, it will be sufficient to prove the following.

Theorem 1. Let $\rho_{A B}$ be an arbitrary state for $A B$. It holds

$$
\sup _{\Lambda^{E B}} F\left(\rho_{A B}, \Lambda_{B}^{E B}\left[\rho_{A B}\right]\right) \geq \sup _{\Lambda^{S y m_{+}(k)}} F\left(\rho_{A B}, \Lambda_{B}^{S y m_{+}(k)}\left[\rho_{A B}\right]\right)-\sqrt{\frac{2|B|}{k}} .
$$

Proof. Let $\Lambda^{\mathrm{Sym}_{+}(k)}$ be a $k$-Bose-extendible channel. Lemma 1 says that there is an entanglement-breaking channel $\Lambda^{\mathrm{EB}}$ such that

$$
\sup _{\rho_{A B}} \Delta\left(\Lambda_{B}^{\mathrm{Sym}_{+}(k)}\left[\rho_{A B}\right], \Lambda_{B}^{\mathrm{EB}}\left[\rho_{A B}\right]\right) \leq \frac{|B|}{k} .
$$

This implies that, for any $\rho_{A B}$ and for any $\Lambda^{\operatorname{Sym}_{+}{ }^{(k)}}$ on $B$, there is $\Lambda^{\mathrm{EB}}$ such that

$$
\Delta\left(\Lambda_{B}^{\mathrm{Sym}_{+}(k)}\left[\rho_{A B}\right], \Lambda_{B}^{\mathrm{EB}}\left[\rho_{A B}\right]\right) \leq \frac{|B|}{k} .
$$

Thus, using Lemma 2, we obtain

$$
\begin{aligned}
F\left(\rho_{A B}, \Lambda_{B}^{\mathrm{EB}}\left[\rho_{A B}\right]\right) \geq & F\left(\rho_{A B}, \Lambda_{B}^{\mathrm{Sym}_{+}(k)}\left[\rho_{A B}\right]\right) \\
& -\sqrt{2} \sqrt{\Delta\left(\Lambda_{B}^{\mathrm{Sym}_{+}(k)}\left[\rho_{A B}\right], \Lambda_{B}^{\mathrm{EB}}\left[\rho_{A B}\right]\right)} \\
\geq & F\left(\rho_{A B}, \Lambda_{B}^{\mathrm{Sym}_{+}(k)}\left[\rho_{A B}\right]\right)-\sqrt{\frac{2|B|}{k}} .
\end{aligned}
$$

Since this is valid for any $\Lambda^{\mathrm{Sym}_{+}(k)}$, we can take the supremum on both sides over channels in the respective classes.

\section{PROPERTIES OF THE HIERARCHY OF DISCORD-LIKE QUANTIFIERS BASED ON LOCAL BROADCASTING}

We argue that $\sup _{\Lambda^{\mathrm{Sym}_{+}(k)}} F\left(\rho_{A B}, \Lambda_{B}^{\mathrm{Sym}_{+}{ }^{(k)}}\left[\rho_{A B}\right]\right)$ can be expressed as

$$
\begin{array}{ll}
\max & \frac{1}{2}\left(\operatorname{Tr}(X)+\operatorname{Tr}\left(X^{\dagger}\right)\right) \\
\text { s. t. } & \left(\begin{array}{cc}
\rho_{A B} & X \\
X^{\dagger} & \operatorname{Tr}_{\backslash A B_{1}}\left(W_{B B^{k}}^{\Gamma_{B}} \rho_{A B}\right)
\end{array}\right) \geq 0 \\
& W_{B B^{k}} \geq 0, W_{B}=\mathbb{1}_{B}, W_{B B^{k}}=\Pi_{B^{k}}^{+} W_{B B^{k}} \Pi_{B^{k}}^{+} .
\end{array}
$$

Two elements will be important in the argument: the expression of the quantum fidelity as SDP, and the ChoiJamiołkowski isomorphism.

We recall that the the fidelity corresponds to the SDP optimization $[5,6]$

$$
\begin{array}{cl}
\text { maximize } & \frac{1}{2}\left(\operatorname{Tr}(X)+\operatorname{Tr}\left(X^{\dagger}\right)\right), \\
\text { subject to } & \left(\begin{array}{cc}
\rho & X \\
X^{\dagger} & \sigma
\end{array}\right) \geq 0
\end{array}
$$

The Choi-Jamiołkowski isomorphism $[7,8]$ is a one-to-one correspondence between linear maps $\Lambda_{X \rightarrow Y}$ from $L\left(\mathcal{H}_{X}\right)$ to $L\left(\mathcal{H}_{Y}\right)$ and linear operators $W_{X Y}$ in $L\left(\mathcal{H}_{X} \otimes \mathcal{H}_{Y}\right)$. It reads

$$
J(\Lambda)_{X Y}=\left(\operatorname{id}_{X} \otimes \Lambda_{X^{\prime} \rightarrow Y}\right)\left[\tilde{\psi}_{X X^{\prime}}^{+}\right],
$$


with inverse (defined by its action on an arbitrary $\rho_{X}$ )

$$
\left(J^{-1}\left(W_{X Y}\right)\right)_{X \rightarrow Y}\left[\rho_{X}\right]=\operatorname{Tr}_{X}\left(W_{X Y}^{\Gamma_{X}} \rho_{X}\right) .
$$

Here $\tilde{\psi}_{X X^{\prime}}^{+}=\left|\tilde{\psi}^{+}\right\rangle\left\langle\left.\tilde{\psi}^{+}\right|_{X X^{\prime}} \text {, with } \mid \tilde{\psi}^{+}\right\rangle_{X X^{\prime}}=\sum_{x}|x\rangle_{X}|x\rangle_{X^{\prime}}$, for $\{|x\rangle\}$ an orthonormal basis of $\mathcal{H}_{X}$, while ${ }^{\Gamma_{X}}$ denotes partial transposition on $X$. The operator $J(\Lambda)$ encodes all properties of the map $\Lambda$. In particular, the linear map $\left(J^{-1}\left(W_{X Y}\right)\right)_{X \rightarrow Y}$ is a quantum channel from $X$ to $Y$ if and only if $W_{X Y} \geq 0$ and $W_{X}=\operatorname{Tr}_{Y}\left(W_{X Y}\right)=\mathbb{1}_{X}$. In addition, $\left(J^{-1}\left(W_{X Y}\right)\right)_{X \rightarrow Y}$ is (i) an entanglement-breaking channel if and only if $W_{X Y}$ satisfies the extra condition of being proportional to a separable state, and (ii) a $k$-Bose-symmetric extendible channel if and only if $W_{X Y}$ admits $k$-Bose-symmetric extensions on $Y$.

The Choi-Jamiołkoski isomorphism allows us to write the fidelity between $\rho=\rho_{A B}$ and $\sigma=\left(\operatorname{id}_{A} \otimes \Lambda_{B}^{\operatorname{Sym}_{+}(k)}\right)\left[\rho_{A B}\right]$, optimized over $\Lambda_{B}^{\mathrm{Sym}_{+}(k)} \in \mathcal{L}^{\mathrm{Sym}_{+}(k)}$, as an optimization over positive semidefinite operators $W_{B B^{k}}$ isomorphic to $k$-Bose-symmetric broadcasting channels, i.e., such that $\operatorname{Tr}_{\backslash B B_{1}}\left(W_{B B^{k}}^{\Gamma_{B}}\right)$ is isomorphic to a $k$-Bose-extendible channel. Thus, adapting Eq. (8), we find that $\sup _{\Lambda \in \mathcal{L}^{\operatorname{Sym}(\mathrm{k})}} F\left(\rho_{A B}, \Lambda_{B}\left[\rho_{A B}\right]\right)$, corresponds to the solution of the SDP (7). As mentioned in the main text, Eqs. (7a) and (7b) directly correspond to Eqs. (8a) and (8b), respectively.

We already argued that $D_{F, \mathcal{L}^{\mathrm{Sym}_{+}(k)}}(A: \underline{B})$ converges to $D_{F, \mathcal{L}^{\mathrm{EB}}}(A: \underline{B})$. To see that it does so monotonically, i.e., that $D_{F, \mathcal{L}^{\mathrm{Sym}(k+1)}}(A: \underline{B}) \geq D_{F, \mathcal{L}^{\mathrm{Sym}_{+}(k)}}(A: \underline{B})$, we notice that, if $W_{B B^{k+1}}$ is Bose-symmetric on $B^{k+1}$, then $\operatorname{Tr}_{B_{k+1}}\left(W_{B B^{k+1}}\right)$ is Bose-symmetric on $B^{k}$. Thus, any feasible $W_{B B^{k+1}}$ for the $k+1$-th element of the SDP hierarchy has a corresponding $W_{B B^{k}}^{\prime}=\operatorname{Tr}_{B_{k+1}}\left(W_{B B^{k+1}}\right)$ that is feasible for the $k$-th element of the hierarchy, with equal achieved fidelity. This proves that the fidelity of transmission at the $k$-th level can only be greater or equal to that at the $k+1$-th level.

Thanks to the monotonicity of the fidelity $F$ under quantum operations, i.e., $F(\Lambda[\sigma], \Lambda[\rho]) \geq F(\sigma, \rho)[1]$, each $D_{F, \mathcal{L}^{\mathrm{Sym}_{+}(k)}}(A: \underline{B})$ is invariant under local unitaries on $B$, and monotonically decreasing under general local operations on $A$. A detailed proof for $D_{F, \mathcal{L}^{\mathrm{EB}}}$, which can be immediately adapted to $D_{F, \mathcal{L}^{\mathrm{Sym}_{+}(k)}}$, is presented in [9]. Thus, each $D_{F, \mathcal{L}^{\mathrm{Sym}_{+}(k)}}$ constitutes a well-behaved measure of the general quantumness of correlations $[10,11]$.

Simply by asking that $W_{B B^{k}}$ in (7) is PPT with respect to the $B: B^{k}$ partition, i.e., $W_{B B^{k}}^{\Gamma_{B}} \geq 0$, we make the corresponding $k$-Bose-extendible channel PPT binding [12], i.e., such that the state $\left(\mathrm{id}_{A} \otimes \Lambda_{B}\right)\left[\sigma_{A B}\right]$ is PPT for all $\sigma_{A B}$. This is a non-trivial constraint even for $k=1$, and, in the case $B$ is a qubit, enough to make the channel entanglement-breaking [13], so that the SDP provides exactly the surprisal of measurement recoverability.

[1] M. A. Nielsen and I. L. Chuang, Quantum computation and quantum information (Cambridge University Press, 2010).

[2] C. A. Fuchs and J. Van De Graaf, Information Theory, IEEE Transactions on 45, 1216 (1999).

[3] G. Chiribella, in Theory of Quantum Computation, Communication, and Cryptography (Springer, 2011), vol. 6519, pp. $9-25$.

[4] A. Uhlmann, Rep. Math. Phys. 9, 273 (1976).

[5] N. Killoran, Ph.D. thesis, University of Waterloo (2012).

[6] J. Watrous, arXiv preprint arXiv:1207.5726 (2012).

[7] M.-D. Choi, Lin. Alg. Appl. 10, 285 (1975).

[8] A. Jamiołkowski, Rep. Math. Phys. 3, 275 (1972).

[9] K. P. Seshadreesan and M. M. Wilde, Phys. Rev. A 92, 042321 (2015), URL http://link.aps.org/doi/10.1103/ PhysRevA.92.042321.

[10] A. Brodutch and K. Modi, Quant. Inf. Comp. 12, 0721 (2012).

[11] M. Piani, Phys. Rev. A 86, 034101 (2012).

[12] P. Horodecki, M. Horodecki, and R. Horodecki, J. Mod. Opt. 47, 347 (2000).

[13] M. Horodecki, P. Horodecki, and R. Horodecki, Phys. Lett. A 223, 1 (1996). 